\title{
COMPLETELY DECOMPOSABLE FLAT MODULES OVER LOCALLY FACTORIAL DOMAINS ${ }^{1}$
}

\author{
E. L. LADY
}

\begin{abstract}
Rank 1 flat modules are classified for a locally factorial noetherian domain by extending the concept of divisor. A direct sum of rank one flat modules in called completely decomposable. A summand of a completely decomposable module is a direct sum of homogeneous components but need not be completely decomposable.
\end{abstract}

Let $R$ be a noetherian domain such that $R_{\mathrm{m}}$ is factorial for each maximal ideal $\mathrm{m}$ and let $Q$ be the quotient field of $R$. Then $R$ is a Krull domain. If $G$ is a torsion free $R$-module, we will write $Q G=Q \otimes G$ and identify $G$ as a submodule of $Q G$. The rank of $G$ is defined to be the dimension of $Q G$. In particular, rank 1 torsion free modules are isomorphic to submodules of $Q$. A direct sum of rank 1 flat modules will be called completely decomposable.

In this paper we will show that some of the theory of completely decomposable torsion free abelian groups $[4, \S \S 85$ and 86$]$ carries over to completely decomposable $R$-modules. In particular, we get a complete description of rank 1 flat modules.

1. Rank 1 flat modules. Let $\mathcal{H}$ be the set of height one prime ideals in $R$. If $G$ is a flat module and $g \in Q G$ and $\mathfrak{p} \in \mathcal{H}$, we write $\operatorname{ord}_{\mathfrak{p}}(g ; G)$ to denote the largest integer $n$ (possibly negative) such that $g \in \mathfrak{p}^{n} G_{\mathfrak{p}}$. We set $\operatorname{ord}_{\mathfrak{p}}(g ; G)=\infty$ if $g \in \mathfrak{p}^{n} G$ for all $n$. (Since $R_{\mathfrak{p}}$ is a discrete valuation ring, it is always true that $g \in \mathfrak{p}^{n} G_{\mathfrak{p}}$ for at least one $n$.) If $G$ is projective and $0 \neq g \in Q G$, then $\operatorname{ord}_{\mathfrak{p}}(g ; Q G)$ is finite for all $\mathfrak{p}$ and zero for almost all $\mathfrak{p}$. (It suffices to consider the case where $G$ is free, and this reduces to the case $G=R$, and then the assertion is simply part of the definition of a Krull domain.)

Recall that a lattice is a finitely generated torsion free $R$-module. A lattice $G$ is divisorial if $G=\cap_{\mathscr{K}} G_{\mathfrak{p}}$. (We identify $G_{\mathfrak{p}}$ as a submodule of $Q G$.) A lattice in $Q$ is also called a fractional ideal. Projective lattices are always divisorial. Conversely, the condition that $R$ be locally factorial is equivalent to saying that all divisorial fractional ideals are projective [2, VII, §3, Proposition 1, p. 503]. However, this is not the case for divisorial lattices with rank larger than 1.

Standard facts about Krull domains, lattices and so forth can be found in [2] or [3].

Received by the editors February 10, 1975.

AMS (MOS) subject classifications (1970). Primary 13C10; Secondary 20K 15.

Key words and phrases. Divisor, type, completely decomposable module, flat, Krull domain.

1 This research was supported by the National Science Foundation contract number GP41984 . 
Proposition 1. If $G$ is a flat module, then $G=\cap_{\mathcal{K}_{\mathrm{C}}} G_{\mathfrak{p}}$. In other words, if $x \in Q G$, then $x \in G$ if and only if $\operatorname{ord}_{\mathfrak{p}}(x ; G) \geqslant 0$ for all $\mathfrak{p} \in \mathcal{H}$.

Proof. If $G$ is free, this is clear (since $R$ is a Krull domain). In general, $G$ is a direct limit of finitely generated free modules $F_{n}$ [6]. Let $x \in \cap{ }_{\mathcal{K}} G_{\mathrm{p}}$. Since $x \in Q G$, then $r x \in G$ for some $r \in R$. Since there are only finitely many $\mathfrak{p} \in \mathscr{H}$ containing $r$ and localization commutes with direct limits, we can find $n$ such that $r x \in \phi_{n}\left(F_{n}\right)$ and $x \in \phi_{n}\left(F_{n}\right)_{\mathfrak{p}}$ for all $\mathfrak{p}$ containing $r$, where $\phi_{n}$ is the canonical map from $F_{n}$ into $G$. Now since $F_{n}$ has finite rank, we can choose $m \geqslant n$ so that the restriction of $\phi_{m}$ to the image of $F_{n}$ in $F_{m}$ is monic. Then $r x=\phi_{m}(r f)$ where $r f \in F_{m}$ and $f \in\left(F_{m}\right)_{\mathfrak{p}}$ for all $\mathfrak{p} \in \mathcal{H}$ containing $r$. It now follows that $f \in \bigcap_{\mathcal{K}}\left(F_{m}\right)_{\mathfrak{p}}=F_{m}$ and so $x=\phi_{m}(f) \in G$.

Corollary 2. Let $B$ be a nonzero submodule of $Q$. Then $B$ is flat if and only if $B=\bigcap_{\mathcal{K}} B_{\mathfrak{p}}$.

Proof. We have the necessity. For the sufficiency, we may as well suppose $R \subseteq B$. For every integer valued function $\chi$ on $\mathcal{H}$ with finite support, let $B(\chi)=\cap_{\mathcal{K}}\left(B_{\mathfrak{p}} \cap \mathfrak{p}^{-\chi(\mathfrak{p})} R_{\mathfrak{p}}\right)$. Each such $B(\chi)$ is a divisorial, hence projective, fractional ideal [2, VII, $\S 3$, Theorem 3, p. 521] and the directed union of the $B(\chi)$ is $\cap{ }_{\mathcal{K}} B_{\mathfrak{p}}$. Hence the latter is flat.

REMARK. Aside from the applications to be made in this paper, the real significance of Theorem 1 and Corollary 2 is that every rank 1 flat module is a directed union of projective fractional ideals.

Corollary 3. Let $G$ be a finite rank flat module and let $B$ be a rank 1 submodule of $G$ such that $G / B$ is torsion free. Then $B$ is flat.

Proof. We identify $Q B$ as a subspace of $Q G$. By Proposition $1, \cap_{{ }_{\mathcal{H}}} B_{\mathfrak{p}}$ $\subseteq G$. But since $G / B$ is torsion free, this implies that $\cap{ }_{\mathcal{K}} B_{\mathfrak{p}}=B$, so $B$ is flat by Corollary 2 .

LEMMA 4. Let $B$ be a rank 1 flat module and $G$ be any flat module. Let $b$ and $g$ be nonzero elements of $B$ and $G$. Then there exists $\phi \in \operatorname{Hom}(B, G)$ such that $\phi(b)=g$ if and only if $\operatorname{ord}_{\mathfrak{p}}(b ; B) \geqslant \operatorname{ord}_{\mathfrak{p}}(g ; G)$ for all $\mathfrak{p} \in \mathcal{H}$.

Proof. The necessity is clear. Now in any case there exists a unique $\phi$ : $Q B \rightarrow Q G$ such that $\phi(b)=g$. We must show that the condition given implies that $\phi(B) \subseteq G$. Now if $x \in B$, then $x=r b$ for some $r \in Q$. Since $R_{\mathfrak{p}}$ is a discrete valuation ring, it is easy to see that

$$
\begin{aligned}
\operatorname{ord}_{\mathfrak{p}}(\phi(x) ; G) & =\operatorname{ord}_{\mathfrak{p}}(g ; G)+\operatorname{ord}_{\mathfrak{p}}(r ; R) \geqslant \operatorname{ord}_{\mathfrak{p}}(b ; B)+\operatorname{ord}_{\mathfrak{p}}(r ; R) \\
& =\operatorname{ord}_{\mathfrak{p}}(r b ; B) \geqslant 0 \quad \text { for all } \mathfrak{p} .
\end{aligned}
$$

Hence $\phi(x) \in G$ by Proposition 1 .

We are now ready to classify rank 1 flat modules.

Definition. An extended divisor is a function $D$ from ith to $\mathbf{Z} \cup\{-\infty\}$ which is nonpositive almost everywhere. A proper divisor is an extended divisor which is finite everywhere and zero almost everywhere. A divisor $D$ is principal if there exists $r \in Q$ such that $D(\mathfrak{p})=\operatorname{ord}_{\mathfrak{p}}(r ; R)$ for all $\mathfrak{p}$. A characteristic is a function from $\mathscr{K}$ to $\mathbf{Z} \cup\{\infty\}$ which is nonnegative almost everywhere. (Thus if $\delta$ is an extended divisor, $-\delta$ is a characteristic.) 
The set $\mathbf{E}$ of extended divisors and the set $\mathbf{C}$ of characteristics are ordered semigroups, and the set $\mathbf{D}$ of proper divisors is a subgroup of each, and the set $\mathbf{P}$ of principal divisors is a subgroup of $\mathbf{D}$. An element of $\mathbf{E} / \mathbf{P}$ is called an extended divisor class and an element of $\mathbf{C} / \mathbf{D}$ is called a type.

THEOREM 5. There is an isomorphism between the multiplicative semigroup of flat submodules of $Q$ and $\mathbf{E}$ given as follows: $A$ flat submodule $B$ of $Q$ corresponds to the divisor $\mathfrak{D}=\operatorname{div} B$ such that $\delta(\mathfrak{p})=-\operatorname{ord}_{\mathfrak{p}}(1 ; B)$ for all $\mathfrak{p}$. In this correspondence, proper divisors correspond to projective fractional ideals and principal divisors correspond to principal fractional ideals.

Proof. Let $B$ be a flat submodule of $Q$ and $\mathfrak{d}=\operatorname{div} B$. For any $\mathfrak{p} \in \mathcal{H}$, since $R_{\mathfrak{p}}$ is a discrete valuation ring, it is easy to see that $B_{\mathfrak{p}}=\mathfrak{p}^{\mathfrak{d}(\mathfrak{p})} R_{\mathfrak{p}}$, where $\mathfrak{p}^{-\infty} R_{\mathfrak{p}}=Q$. It is now clear that div is an epimorphism, and from Corollary 2 we see that it is a monomorphism. It is also easy to check locally that $\operatorname{div}(B C)=\operatorname{div} B+\operatorname{div} C$. The last statement of the theorem is classical [3].

Definition. Two finite rank torsion free modules $G$ and $H$ are quasiisomorphic if there exist monomorphisms from $G$ to $H$ and from $H$ to $G$.

REMARK. If $G$ and $H$ are finite rank torsion free modules, we can define the group of quasi-homomorphisms from $G$ to $H$ to be $Q \otimes \operatorname{Hom}(G, H)$. Quasiisomorphism, as defined above, amounts to isomorphism in the resulting category. The usefulness of this category arises from the fact that in it objects have unique direct sum decompositions (up to isomorphism). Both these assertions follow easily from the fact that $Q \otimes$ End $G$ is a finite dimensional $Q$-algebra. For the benefit of the reader unfamiliar with quasi-isomorphism, we give the following lemma:

LEMMA 6. Two finite rank torsion free modules $G$ and $H$ are quasi-isomorphic if and only if there is a submodule $G^{\prime} \subseteq G$ such that $G^{\prime} \approx H$ and $G / G^{\prime}$ is not faithfül.

Proof. The sufficiency is easy. The necessity follows if we can show that whenever $\phi$ is a monomorphism in End $G$, then $r(G / \phi G)=0$ for some nonzero $r \in R$. But $\phi$ has an inverse $\psi \in Q \otimes$ End $G$, since it is not a zero divisor. Now choose $r$ so that $r \psi \in$ End $G$.

If $B$ is any rank 1 torsion free module, we let $B^{*}=\bigcap_{{ }_{\mathcal{C}}} B_{p}$. By Corollary 2, $B^{*}$ is the smallest flat submodule of $Q B$ containing $B$.

THEOREM 7. The correspondence in Theorem 5 induces a 1-1 correspondence between isomorphism classes of rank 1 flat modules and extended divisor classes, and also (via an appropriate minus sign) between quasi-isomorphism classes of rank 1 modules and types. The ordering in $\mathbf{C}$ induces an ordering on $\mathbf{C} / \mathbf{D}$. If $\mathbf{t}(B)$ denotes the type corresponding to $-\operatorname{div} B$, then $\mathbf{t}(B) \leqslant \mathbf{t}(C)$ if and only if $\operatorname{Hom}(B, C) \neq 0$. The set of types $\mathbf{C} / \mathbf{D}$ is in fact lattice ordered, and

$$
\mathbf{t}(B) \wedge \mathbf{t}(C)=\mathbf{t}(B \cap C), \quad \mathbf{t}(B) \vee \mathbf{t}(C)=\mathbf{t}\left((B+C)^{*}\right)
$$

Proof. Two submodules $B$ and $C$ of $Q$ are isomorphic if and only if $B=r C$ for some nonzero $r \in Q$, and if $B$ and $C$ are flat this is equivalent to saying $\operatorname{div} B-\operatorname{div} C=\operatorname{div}(r R)$ by Theorem 5 . The fact that $\mathbf{t}(B) \leqslant \mathbf{t}(C)$ if and only if $\operatorname{Hom}(B, C) \neq 0$ follows easily from Lemma 4. Since nonzero homomorphisms between rank 1 flat modules are monic, this shows that 
$\mathbf{t}(B)=\mathbf{t}(C)$ if and only if $B$ and $C$ are quasi-isomorphic. The remaining statement is easy, since each $R_{\mathfrak{p}}$ is a discrete valuation ring.

Corollary 8. If $B$ and $C$ are rank 1 flat modules, then $B$ and $C$ are quasi-isomorphic if and only if $C \approx I B$ for some projective fractional ideal $I$.

Proof. In fact,

$$
\mathbf{t}(B)=\mathbf{t}(C) \Leftrightarrow \operatorname{div} B-\operatorname{div} C \in D \Leftrightarrow \operatorname{div} B-\operatorname{div} C=\operatorname{div} I
$$

for some projective fractional ideal $I$.

COROllaRy 9. Quasi-isomorphism coincides with isomorphism for rank 1 flat modules if and only if $R$ is factorial.

Proof. It is well known that $\mathbf{D}=\mathbf{P}$ if and only if $R$ is factorial.

2. Completely decomposable modules. In order to understand completely decomposable modules, we need to be able to associate a type to each element in a flat module.

Definition. If $G$ is a flat module and $0 \neq g \in Q G$, we define $\mathbf{t}(g ; G)$ to be the type represented by the characteristic $\chi$ such that $\chi(\mathfrak{p})=\operatorname{ord}_{\mathfrak{p}}(g ; G)$ for all $\mathfrak{p} \in \mathcal{H}$. We set $\mathbf{t}(0 ; G)=\mathbf{t}(Q)$. Note that $\mathbf{t}(g ; G)=\mathbf{t}(G \cap Q g)$. In particular, if $G$ has rank 1 and $0 \neq g \in G$, then $\mathbf{t}(g ; G)=\mathbf{t}(G)$.

If $G$ is a flat module and $\mathbf{t}$ a type, we set $G(\mathbf{t})=\{g \in G \mid \mathbf{t}(g) \geqslant \mathbf{t}\}$. We say that $G$ is $\mathbf{t}$-homogeneous if $\mathbf{t}(g)=\mathbf{t}$ for all nonzero $g \in G$. A t-homogeneous completely decomposable module will be called $\mathbf{t}$-free. A summand of a $\mathbf{t}$-free module will be called t-projective.

THEOREM 10. Let $G$ be a completely decomposable module. Then $G$ $=\mathrm{II}_{\mathbf{C} / \mathbf{D}} G_{\mathbf{t}}$, where each $G_{\mathbf{t}}$ is $\mathbf{t}$-free. The $G_{\mathbf{t}}$ are unique up to isomorphism. If $G=H \oplus K$, then $H=\amalg H_{\mathrm{t}}, K=\amalg K_{\mathrm{t}}$ where the $H_{\mathrm{t}}$ and $K_{\mathrm{t}}$ are t-projective and $H_{\mathrm{t}} \oplus K_{\mathrm{t}} \approx G_{\mathrm{t}}$.

Proof. Exactly as in abelian group theory [4, Theorem 86.7, p. 114].

REMARK. It is easy to show that if $B$ is a rank 1 flat module and $\mathbf{t}=\mathbf{t}(B)$, then all $\mathbf{t}$-projective modules are $\mathbf{t}$-free if and only if all projective End $B$ modules are free. (See [1, Theorem 1.1] for the basic idea.) Furthermore, End $B=\bigcap\left\{R_{\mathfrak{p}} \mid(\operatorname{div} B)(\mathfrak{p})\right.$ is finite $\}$.

There are two nice theorems in abelian group theory stating that in certain circumstances when $\mathbf{t}$-projective groups are at hand, one can conclude that pure submodules are in fact summands [4, Theorems 86.5 and 86.8, p. 114]. The next proposition is a simultaneous counterexample to both these theorems in case $R$ has dimension larger than 1. We also see that a pure submodule of a t-projective module need not, in general, be t-projective. (Recall that if $G$ is flat, then $H$ is pure in $G$ if and only if $G / H$ is flat.)

Proposition 11. If $R$ has Krull dimension larger than 1 , then there exists $a$ type $\mathbf{t}, a$ t-free module $G$ and a pure submodule $H$ such that $G / H \approx B$ where $B$ has rank 1 and $\mathbf{t}(B)=\mathbf{t}$, and $H$ is not a summand of $G$. Furthermore, unless $R$ is regular with dimension at most 2, we can choose $G$ and $H$ so that $H$ is not t-projective. 
Proof. Choose a prime ideal $m$ with height at least 2 and such that, if possible, $R_{\mathfrak{m}}$ is not a two dimensional regular local ring. Localizing at $\mathrm{m}$, we may as well suppose that $R$ is factorial. By [5, Theorem 144, p. 107] $\mathrm{m}$ contains infinitely many height one primes $\mathfrak{p}$. Now let $\chi$ be the characteristic defined by $\chi(\mathfrak{p})=1$ for all $\mathfrak{p} \in \mathcal{K}$, and let $\mathbf{t}$ be the type corresponding to $\chi$. Choose generators $x_{1}, \ldots, x_{s}$ for $m$ and map $R \oplus \cdots \oplus R$ onto $m$ by $\left(r_{1}, \ldots, r_{s}\right) \rightarrow \sum r_{i} x_{i}$. Choose $B \subseteq Q$ so that $\operatorname{div} B=-\chi$. Now if $b \in B$, then $\operatorname{ord}_{\mathfrak{p}}(b ; B)=1$ for some (in fact infinitely many) $\mathfrak{p} \subseteq \mathrm{m}$. Choose $r \in R$ so that $\mathfrak{p}=(r)$. Then $r^{-1} b \in B$ and $b=r\left(r^{-1} b\right) \in \mathfrak{m} B$. Thus we see that $\mathrm{m} B=B$. Tensoring the surjection $R \oplus \cdots \oplus R \rightarrow \mathrm{m}$ by $B$, we get a pure exact sequence $0 \rightarrow H \rightarrow B \oplus \cdots \oplus B \rightarrow{ }^{\pi} B \rightarrow 0$. If this sequence were split, then applying $\operatorname{Hom}(B, *)$ would give an exact sequence

$$
0 \rightarrow \operatorname{Hom}(B, H) \rightarrow R \oplus \cdots \oplus \stackrel{\pi_{*}^{*}}{\rightarrow} R \rightarrow 0 .
$$

But this is not the case, since it is easily seen that the image of $\pi_{*}$ is $\mathrm{m}$. Furthermore, if $H$ were t-projective, then $\operatorname{Hom}(B, H)$ would be projective. Since Image $\left(\pi_{*}\right)=\mathfrak{m}$, this would mean that $m$ has projective dimension at most 1 , and this cannot happen unless $R_{\mathrm{m}}$ is a two dimensional regular local ring.

\section{REFERENCES}

1. D. M. Arnold and E. L. Lady, Endomorphism rings and direct sums of torsion free abelian groups, Trans. Amer. Math. Soc. 211 (1975), 225-237.

2. N. Bourbaki, Commutative algebra, Addison-Wesley, Reading, Mass., 1972.

3. R. Fossum, The divisor class group of a Krull domain, Springer-Verlag, New York, 1973.

4. L. Fuchs, Infinite abelian groups, Vol. II, Academic Press, New York, 1973.

5. I. Kaplansky, Commutative rings, Univ. of Chicago Press, Chicago, Ill., 1974.

6. D. Lazard, Autour de la platitude, Bull. Soc. Math. France 97(1969), 81-128. MR40 \#7310; erratum, 41, p. 1965.

Department of Mathematics, University of Kansas, Lawrence, Kansas 66045 